\title{
Supernetwork Model of Enterprise Marketing Based on Complex Network Theory
}

\author{
Junquan Gong \\ Business School \\ Tianshui Normal University \\ Tianshui,China \\ 314695600@qq.com
}

\author{
Xiaohong Qin \\ Business School \\ Tianshui Normal University \\ Tianshui,China \\ qinxiaohong12@sohu.com
}

\begin{abstract}
The authors has established the supernetwork model of enterprise marketing in order to study equilibrium on the network of enterprise marketing, and has analysised of manufacturers, intermediate traders and consumers behavior and optimal target. Then the optimal goal has changed into the corresponding variational inequality. The article has achieved the win-win equilibrium state and solution algorithm of supernetwork model of enterprise marketing. Finally, an example has given to validate the effectiveness of the model and solution algorithm.
\end{abstract}

Keywords- enterprise marketing network; supernetwork; variational inequality; supernetwork model; optimal target

\section{INTRODUCTION}

Many marketing channels has formed marketing network with the enterprise marketing environment getting more and more complex. Marketing network is not only of logistics network, but also is value added network. Products is increase the value with it transfer process for processing, packaging, transporting and stocking in marketing channel, and the related member of enterprise marketing network gain benefits. The enterprise marketing network equilibrium has researched in past time, but about its achievements has limited competition and cooperation on same marketing channel, different marketing channels members and different layers members of same marketing channels on different network had not studied. Super-network theory has provides new tools to study the marketing network for difference and same marketing channels in different layer. The supernetwork is a complex network because it included many network, which have multilayer and multi-level characteristics .

In this paper, the authors has established the supernetwork model of enterprise marketing base on the supernetwork theory, then has analysised each layer of marketing network behaviors and optimal targets. Furthermore, The authors has studied win-win balance state and solution algorithm of supernetwork mode of enterprise marketing.

\section{THE CONTROL STRATEGY SELECTION}

\section{SUPERNETWORK MODEL OF ENTERPRISE MARKETING}

Marketing network is a complex system for product supply and demand between manufacturers and intermediate trader (intermediate trader is refer all organizations or individuals of participate in commodity trading which locate in manufactures and consumers between, such as wholesalers and retailers, and so on), and consumers (consumers include organizations and individuals ).Hypothesis enterprise marketing network consists of $m$ manufacturers, $n$ intermediate traders and $O$ consumers, each member is abstract a node, and a side is builted from the supplier to the demander if the two node between exists transaction relationship. According to each member position and division of labor different, thus has obtained a three layer structure supernetwork model of enterprise marketing, as shown in figure 1.

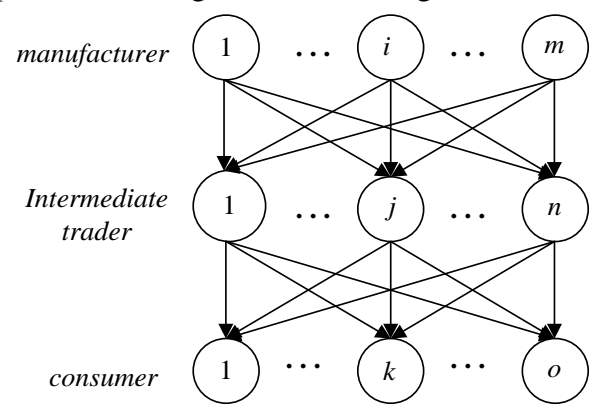

Figure 1.

$$
\text { supernetwork model of enterprise marketing }
$$

In the supernetwork model of enterprise marketing ,this paper hypothesis that manufacturers is product the same or the same brand product which it with the same value; and between adjacent layers can be freely trade, and consumption market state is equilibrium. And following, the author analysis manufacturer and intermediate trader and consumer behavior and its optimization target..

\section{A. Manufacturer behavior and its optimization target}

Choice a manufacturer $i(i=1, \cdots, m)$ as the object. For manufacturer $i$, its production cost function $f_{i}$ is correlation $q_{i}$ In the case of fixed cost, In which $q_{i}$ is intermediate trader to order product from manufacturer $i$. Making $\quad q_{i j}$ express intermediate trader $j(j=1,2, \cdots, n)$ to order products from manufacturer $i$, which is component of $Q^{1} \in R_{+}^{m n}$ a vector. Considering the same layer members competition with each others, $f_{i}$ is correlation with order quantity of other manufacturers . In view of this, we will set $f_{i}$ to:

$$
f_{i}=f_{i}\left(Q^{1}\right), \forall i
$$


And we hypothesis $f_{i}$ is a continuously differentiable non-negative convex function.

In addition, manufacturers $i$ with intermediate trader $j$ transaction has certain costs $c_{i j}$, which is include transport costs, travel costs, and other related charges. It is a function about $i$ with $j$ products trading quantity, $c_{i j}$ as:

$$
c_{i j}=c_{i j}\left(q_{i j}\right), \forall i, j
$$

And we hypothesis $c_{i j}$ is a continuously differentiable non-negative convex function.

With $\rho_{1 i}^{*}$ representation to unit product wholesale price of manufacturer $i$, where the subscript 1 indicates that it is the first layer of the variables. The total cost of $i$ is equal to its production cost and transaction cost. While manufacturers $i$ income is equal to the unit product wholesale price multiply with trading volume. Thus, producers $i$ in the transaction process maximum profit can expressed as:

$$
\max z_{1 i}=\sum_{j=1}^{n} \rho_{1 i}^{*} q_{i j}-\left(Q^{1}\right)-\sum_{j=1}^{n} c_{i j}\left(q_{i j}\right)
$$

For $\forall i=1,2, \cdots, m ; j=, 2, \cdots, n \quad, \quad$ to have $q_{i j} \geq 0$,for all $i=1,2, \cdots, m$, the target function $z_{1 i}$ of manufacturers $i$ is a convex function. In this way, the solution of $z_{1 i}$ is also equal to solution corresponding of variational inequality $\left(Q^{1^{*}}, \eta^{*}\right) \in R_{+}^{m n+m}$ :

$$
\begin{aligned}
& \sum_{i=1}^{m} \sum_{j=1}^{n} \frac{\partial z_{1 i}\left(q_{i j}^{*}, \eta_{i}^{*}\right)}{\partial q_{i j}} \times\left(q_{i j}-q_{i j}^{*}\right) \\
& +\sum_{i=1}^{m} \frac{\partial z_{1 i}\left(q_{i j}^{*}, \eta_{i}^{*}\right)}{\partial \eta_{i}} \times\left(\eta_{i}-\eta_{i}^{*}\right) \geq 0
\end{aligned}
$$

In which $\eta_{i}$ is lagrange factor as $q_{i j} \geq 0$ and $\forall i=1,2, \cdots, m ; j=, 2, \cdots, n . \eta$ is vector of all manufacturer Lagrange factors.

\section{B. Intermediate trader behavior and its optimization target}

Choice a intermediate trader $j(j=1, \cdots, n)$ as the object. For intermediate trader $j$, it needs to pay a certain cost, such as inventory costs, product costs and equipment repair costs. The cost is a function about $\sum_{i=1}^{m} q_{i j}$. same as the manufacturers to optimize behavioral analysis, also considering the intermediate trader between have competing relationship, so, $c_{j}$ with correlation vector $Q^{1}$. We denote:

$$
c_{j}=c_{j}\left(Q^{1}\right), \forall j
$$

And we hypothesis $c_{j}$ is a continuously differentiable non-negative convex function.
The intermediate trader pay certain transaction costs when transactions with manufacturers. This cost is function about the trading quantity, we denote:

$$
\hat{C_{i j}}=\hat{C_{i j}}\left(q_{i j}\right), \forall i, j
$$

And we hypothesis $\boldsymbol{C}_{i j}$ is a continuously differentiable non-negative convex function.

The $r_{j}^{*}$ is price of unit product. The $q_{j k}$ as product quantity which is intermediate $j$ with consumer $k$

$\left(k=1, \cdots, o \quad\right.$ ) ,all $q_{j k}$ composition vector $Q^{2} \in R_{+}^{n o}$.Therefore, the total cost of intermediate trader $j$ is equal the transaction cost and its products processing costs, and its revenue is equal income of sale products to consumer. Thus, intermediate trader $j$ in the transaction process maximum profit can expressed as:

$$
\max z_{2 j}=r_{j}^{*}=\sum_{k=1}^{o} q_{j k}-c_{j}\left(Q^{1}\right)-\sum_{i=1}^{m} \hat{c}_{i j}\left(q_{i j}\right)
$$

For $\forall j=1,2, \cdots, n$, has $\sum_{k=1}^{o} q_{j k} \leq \sum_{i=1}^{m} q_{i j}$ and $q_{i j} \geq 0$.

As the intermediate trader products processing cost function and the function of the transaction costs are continuously differentiable convex function, easy to verify for all $j=1,2, \cdots, n$, the intermediate $j$ target function $z_{2 j}$ is a convex function. In this way, the objective function of the solution is also the corresponding solution of variational inequality $\left(Q^{1^{*}}, Q^{2^{*}}, \rho_{2 j}^{*}\right) \in R_{+}^{m n+n o+n}$ :

$$
\begin{aligned}
& \sum_{i=1}^{m} \sum_{j=1}^{n} \frac{\partial z_{2 j}\left(q_{i j}^{*}, q_{j k}^{*}, \rho_{2 j}^{*}\right)}{\partial q_{i j}} \times\left(q_{i j}-q_{i j}^{*}\right) \\
& +\sum_{j=1}^{n} \sum_{k=1}^{o} \frac{\partial z_{2 j}\left(q_{i j}^{*}, q_{j k}^{*}, \rho_{2 j}^{*}\right)}{\partial q_{j k}} \times\left(q_{j k}-q_{j k}^{*}\right) \\
& +\sum_{j=1}^{n} \frac{\partial z_{2 j}\left(q_{i j}^{*}, q_{j k}^{*}, \rho_{2 j}^{*}\right)}{\partial \rho_{2 j}} \geq 0
\end{aligned}
$$

Among $\rho_{2 j}$ to $\forall j=1,2, \cdots, n$, to have the Lagrange factor of $\sum_{k=1}^{o} q_{j k} \leq \sum_{i=1}^{m} q_{i j}$ and $q_{i j} \geq 0$.

\section{Consumer behavior and its optimization target}

Choice a consumer $k(k=1,2, \cdots, o)$ as the object. $\hat{C_{j k}}$ is transaction cost of consumer $k$ transaction produce with intermediate trader $j$, such as transportation, communication cost..

It is functions related of product trades quantity $q_{j k}$. Using $\rho_{3 k}$ express price of consumer $k$ to need of product, then all demand price composition vector $\rho_{3} \in R_{+}^{o}$, it is a function of:

$$
d_{k}=d_{k}\left(\rho_{3}\right), \quad \forall k
$$


And we hypothesis $d_{k}$ to be continuous and monotone decreasing.

Consumer purchase units product to pay the total cost equal the price of goods and transaction cost. of related. With the consumer $k$ as the case, for $\forall j$ ( $j=1,2, \cdots, n)$, the consumer $k$ price equilibrium conditions to satisfy the following expression:

$$
r_{j}^{*}+\hat{C}_{j k}^{\wedge}\left(q_{j k}^{*}\right)\left\{\begin{array}{l}
=\rho_{3 k}^{*}, q_{j k}^{*}>0 \\
\geq \rho_{3 k}^{*}, q_{j k}^{*}=0
\end{array}\right.
$$

Thus, the consumer $k$ equilibrium conditions can be

$$
d_{k}\left(\rho_{3}^{*}\right)\left\{\begin{array}{l}
=\sum_{j=1}^{n} q_{j k}^{*}, \rho_{3 k}^{*}>0 \\
\leq \sum_{j=1}^{n} q_{j k}^{*}, \rho_{3 k}^{*}=0
\end{array}\right.
$$

expressed:

For $k=1,2, \cdots, o$, consumer optimization target can also be expressed as the form of inequality, in order to solve the balance point $\left(Q^{2^{*}}, \rho_{3 k}^{*}\right) \in R_{+}^{n o+o}$ :

$$
\begin{aligned}
& \sum_{j=1}^{n} \sum_{k=1}^{o}\left(r_{j}^{*}+\hat{\boldsymbol{C}}_{j k}\left(q_{j k}^{*}\right)-\rho_{3 k}^{*}\right) \times\left(q_{j k}-q_{j k}^{*}\right) \\
& +\sum_{k=1}^{o}\left(\sum_{j=1}^{n} q_{j k}^{*}-d_{k}\left(\rho_{3}^{*}\right)\right) \times\left(\rho_{3 k}-\rho_{3 k}^{*}\right) \geq 0
\end{aligned}
$$

III. WIN -WIN EQUILIBRIUM STATE AND SOLUTION ALGORITHM OF SUPERNETWORK MODEL OF ENTERPRISE

\section{MARKETING}

$\left(Q^{1^{*}}, Q^{2^{*}}, \eta^{*}, \rho_{2 j}^{*}, \rho_{3 k}^{*}\right) \in K$ can make manufacturer and intermediate trader and consumer are the greatest extent possible to meet their requirements if Win-win equilibrium state of supernetwork model of enterprise marketing is presence. As existence $\left(Q^{1^{*}}, Q^{2^{*}}, \eta^{*}, \rho_{2 j}^{*}, \rho_{3 k}^{*}\right) \in K$ meet the optimal condition total of (4), (8) and (12).

That is to say the inequality (13) is solvable if win-win equilibrium state of supernetwork model of enterprise marketing can achieve. Reference [4] has proved that the variational inqualities (13) is strictly monotone and have at least one solution.

Equilibrium conditions of supernetwork model of enterprise marketing to meet the existence, and only have one solution.

$$
\begin{aligned}
& \sum_{i=1}^{m} \sum_{j=1}^{n} \frac{\partial z_{1 i}\left(q_{i j}^{*}, \eta_{i}^{*}\right)}{\partial q_{i j}} \times\left(q_{i j}-q_{i j}^{*}\right) \\
& +\sum_{i=1}^{m} \frac{\partial z_{1 i}\left(q_{i j}^{*}, \eta_{i}^{*}\right)}{\partial \eta_{i}} \times\left(\eta_{i}-\eta_{i}^{*}\right) \\
& +\sum_{i=1}^{m} \sum_{j=1}^{n} \frac{\partial z_{2 j}\left(q_{i j}^{*}, q_{j k}^{*}, \rho_{2 j}^{*}\right)}{\partial q_{i j}} \times\left(q_{i j}-q_{i j}^{*}\right) \\
& +\sum_{j=1}^{n} \sum_{k=1}^{o} \frac{\partial z_{2 j}\left(q_{i j}^{*}, q_{j k}^{*}, \rho_{2 j}^{*}\right)}{\partial q_{j k}} \times\left(q_{j k}-q_{j k}^{*}\right) \\
& +\sum_{j=1}^{n} \frac{\partial z_{2 j}\left(q_{i j}^{*}, q_{j k}^{*}, \rho_{2 j}^{*}\right)}{\partial \rho_{2 j}} \\
& +\sum_{j=1}^{n} \sum_{k=1}^{o}\left(r_{j}^{*}+\hat{\boldsymbol{C}}_{j k}\left(q_{j k}^{*}\right)-\rho_{3 k}^{*}\right) \times\left(q_{j k}-q_{j k}^{*}\right) \\
& +\sum_{k=1}^{o}\left(\sum_{j=1}^{n} q_{j k}^{*}-d_{k}\left(\rho_{3}^{*}\right)\right) \times\left(\rho_{3 k}-\rho_{3 k}^{*}\right) \geq 0
\end{aligned}
$$

Now we has given algorithm of win-win equilibrium state of super- network model of enterprise marketing as following.

initialization $\left(Q^{1^{*}}, Q^{2^{*}}, \eta^{*}, \rho_{2 j}^{*}, \rho_{3 k}^{*}\right) \in K$, to make

\section{$T=1$ and to set step $\alpha$.}

Iterative calculation:

Calculation of intermediater to manufacturer product purchases quantity and to consumer product sales quantity from Iterative calculation $\left(\bar{Q}^{1^{T}}, \bar{Q}^{2^{T}}, \bar{\eta}^{T}, \bar{\rho}_{2 j}^{T}, \bar{\rho}_{3 k}^{T}\right) \in K$

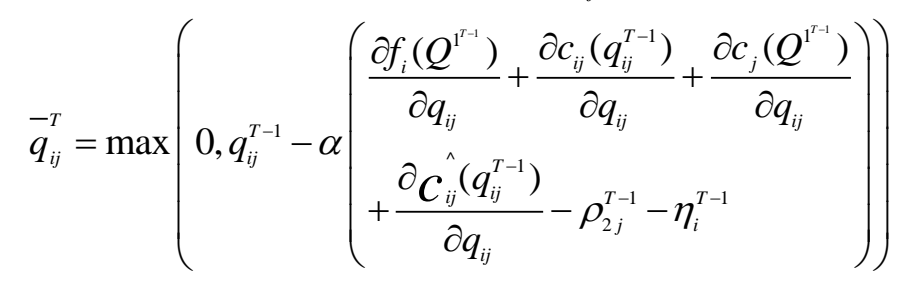

$$
\bar{q}_{j k}^{T}=\max \left(0, q_{j k}^{T-1}-\alpha\left[\rho_{2 j}^{T-1}+\hat{c}_{j k}\left(q_{j k}^{T-1}\right)-\rho_{3 k}^{T-1}\right]\right)
$$

the total cost of $\bar{\rho}_{3 k}^{T}$ which is consumer pay for the units product:

$$
\bar{\rho}_{3 k}^{T}=\max \left(0, \bar{\rho}_{3 k}^{T}-\alpha\left(\sum_{j=1}^{n} q_{j k}^{T-1}-d_{k}\left(\rho_{3}^{T-1}\right)\right)\right)
$$

Lagrange multiplier $\bar{\eta}_{i}^{T} 、 \bar{\rho}_{2 j}^{T}$ as following:

$$
\begin{aligned}
& \bar{\eta}_{i}^{T}=\max \left(0, \eta_{i}^{T-1}-\alpha \times \sum_{j=1}^{n} q_{j k}^{T-1}\right) \\
& \bar{\rho}_{2 j}^{T}=\max \left(0, \rho_{2 j}^{T-1}-\alpha\left(\sum_{i=1}^{m} q_{i j}^{T-1}-\sum_{k=0}^{o} q_{j k}^{T-1}\right)\right)
\end{aligned}
$$

Iterative calculation $\left(Q^{1^{T}}, q^{2^{T}}, \eta^{T}, \rho_{2 j}^{T}, \rho_{3 k}^{T}\right) \in K$

$$
\begin{aligned}
& \text { For any } \varepsilon>0 \text { until } \\
& \left|q_{i j}^{T}-q_{i j}^{T-1}\right|<\varepsilon \quad, \quad\left|q_{j k}^{T}-q_{j k}^{T-1}\right| \leq \varepsilon \quad, \quad\left|\rho_{2 j}^{T}-\rho_{2 j}^{T-1}\right| \leq \varepsilon \quad \text {, }
\end{aligned}
$$


$\left|\rho_{3 k}^{T}-\rho_{3 k}^{T-1}\right| \leq \varepsilon,\left|\rho_{3 k}^{T}-\rho_{3 k}^{T-1}\right| \leq \varepsilon,\left|\eta_{i}^{T}-\eta_{i}^{T-1}\right| \leq \varepsilon$ $(i=1, \cdots, m, j=1, \cdots, n, \quad k=1, \cdots, o$ ) to exit the loop, otherwise, to make $T=T+1$, returning to the step (2) continued iteration.

Such this, we can use the algorithm to achieve Winwin equilibrium state $\left(Q^{1^{*}}, Q^{2^{*}}, \eta^{*}, \rho_{2 j}^{*}, \rho_{3 k}^{*}\right) \in K$. Thus obtained the manufacturer wholesale price $\rho_{1 i}^{*}$ and intermediate trader total distribution quantity $r_{j}^{*}$ is:

$$
\rho_{1 i}^{*}=\frac{\partial f_{i}\left(Q^{1 *}\right)}{\partial q_{i j}}+\frac{\partial c_{i j}\left(q_{i j}^{*}\right)}{\partial q_{i j}}, \quad r_{j}^{*}=\rho_{2 j}^{*}, \forall j
$$

\section{THE EXAMPLE ANALYSIS AND DISCUSSION}

To construct a supermarket model which composed of two producers , two intermediate and two consumer.

The production function of producers set:

$$
f_{1}(q)=2.5 q_{1}^{2}+3 q_{1} ; f_{2}(q)=2.5 q_{2}^{2}+q_{1} q_{2}
$$

Among them, $q_{1}=q_{11}+q_{12} ; q_{2}=q_{21}+q_{22}$

Transaction costs function between producers and middlemen is set to:

$$
\begin{aligned}
& c_{11}\left(q_{11}\right)=0.5 q_{11}^{2}+3.5 q_{11}, \\
& c_{12}\left(q_{12}\right)=0.5 q_{12}^{2}+3.5 q_{12} \\
& c_{21}\left(q_{21}\right)=0.5 q_{21}^{2}+3.5 q_{21}, \\
& c_{22}\left(q_{22}\right)=0.5 q_{22}^{2}+3.5 q_{22}
\end{aligned}
$$

Commodity display and storage cost function of middlemen is set to:

$$
\begin{aligned}
& c_{1}\left(Q^{1}\right)=0.5\left(q_{11}+q_{21}\right)^{2}, \\
& c_{2}\left(Q^{1}\right)=0.5\left(q_{12}+q_{22}\right)^{2}
\end{aligned}
$$

Transaction costs function between brokers and manufacturers set:

$$
\begin{aligned}
& \hat{\boldsymbol{c}}_{11}\left(q_{11}\right)=0.5 q_{11}^{2}+3 q_{11}, \\
& \hat{\boldsymbol{c}}_{12}\left(q_{12}\right)=0.5 q_{12}^{2}+3 q_{12} \\
& \hat{\boldsymbol{c}}_{21}\left(q_{21}\right)=0.5 q_{21}^{2}+3 q_{21}, \\
& \hat{\boldsymbol{c}}_{22}\left(q_{22}\right)=0.5 q_{22}^{2}+3 q_{22}
\end{aligned}
$$

The intermediary and final consumption market transaction cost is set to:

$$
\begin{array}{ll}
c_{11}\left(q_{11}\right)=q_{11}+1, & c_{12}\left(q_{12}\right)=q_{12}+1 \\
c_{21}\left(q_{21}\right)=q_{21}+1, & c_{22}\left(q_{22}\right)=q_{22}+1
\end{array}
$$

Consumer demand functions set:

$$
\begin{aligned}
& d_{1}\left(\rho_{3}\right)=-2 \rho_{31}-1.5 \rho_{32}+1000 \\
& d_{2}\left(\rho_{3}\right)=-2 \rho_{32}-1.5 \rho_{31}+1000
\end{aligned}
$$

Parameter is set to:

$$
\begin{gathered}
\varepsilon=10^{-3}, \alpha=0.005, \alpha_{1}=\alpha_{2}=0.03, \\
\beta_{1}=\beta_{2}=0.02
\end{gathered}
$$

Using Matlab simulation, 500 iterations are middlemen to optimal product manufacturers ordering between trading volume weight, middlemen and consumers are as follows:

Middlemen to order quantity production for:

$$
\begin{aligned}
& q_{11}^{*}=16.28, q_{12}^{*}=14.96, \\
& q_{21}^{*}=14.52, q_{22}^{*}=17.58
\end{aligned}
$$

Products trade volume of consumers and intermediaries for:

$$
\begin{aligned}
& q_{11}^{*}=15.36, q_{12}^{*}=15.36, \\
& q_{21}^{*}=16.25, q_{22}^{*}=16.25
\end{aligned}
$$

Consumers pay for the unit price of the product is:

$$
\rho_{31}^{*}=276.70, \rho_{32}^{*}=276.70
$$

In addition, by formula (4) and (8) the wholesale price and middlemen to production of products for the retail price:

$$
\begin{gathered}
\rho_{11}=209.83, \rho_{12}=211.80, r_{1}=260.24, \\
r_{2}=259.36
\end{gathered}
$$

\section{CONLUSIONS}

This paper has established the supernetwork model of enterprise marketing, and has obtained the marketing network of producers, middlemen and consumers behavior and optimal conditions, and then studied the enterprise marketing super network model of win-win equilibrium state, and an example is used to verify the validity of the model and algorithm. The conclusion of the study on enterprise marketing network each nets member decision to provide a practical reference is of great value. Establishment with three or more layers supernetwork model of enterprise marketing is the further research direction.

\section{REFERENCES}

[1] Zhang Xinrui, the king of Mt. Hengshan. The complex supply chain network structure model $[\mathrm{J}]$. Industrial technology economy,2008(2):65-70.

[2] Zhang Xinrui, Wang Mt. Hengshan. Supply chain network value added game decision making research [J ]. Business Economics and management, 2009(7):70-81.

[3] Wang Zhiping, Wang Zhong. The network theory and its application [ J]. Beijing: Science press, 2008.

[4] David K L, Guido S. An introduction to variational inequalities and their applications. Academic Press, 1980.

[5] Fleischmann M, etal. Controlling inventories with stochastic item return: a basic model. European Journal of Operational Research, 2002,138:62-75.

[6] Zhang Tiezhu, Hu Yunquan, and so on. Decentralized supply chain planning model for multilayer [ $\mathrm{J}$ ]. Heilongjiang University Natural Science Journal,2006,23(3):302-306.

[7] Susan A Sherer. From Supply-chain Management to Value Network Advocacy: Implications for E-supply Chains[J].Supply Chain Management,2005,10(2):7 